\title{
A Pilot Study Examining the Effect of Acceptance and Commitment Therapy as Parent Counseling
}

\author{
Denise H. M. Bodden $\mathbb{1}^{1,2} \cdot$ Denise Matthijssen ${ }^{2}$
}

Accepted: 14 February 2021 / Published online: 27 February 2021

(c) The Author(s) 2021

\begin{abstract}
There is some scientific evidence to support the applicability and preliminary effects of ACT as a parent intervention but more research is needed. In this pilot research, Acceptance and Commitment Therapy (ACT) is provided as a parent counseling therapy in order to increase psychological flexibility which in turn helps parents to choose attuned parenting behavior, invest in a helpful parent-child relationship, deal with feelings of incompetence, and cope with their own psychopathology. ACT parent counseling is developed for parents of children (3-18 years old) with psychiatric problems. The mean age of the parents $(n=101)$ was 47.1 years (range $=30-66)$. A repeated measures design was used including pretreatment, posttreatment, and 6-month follow-up measurements. The main outcomes were ACT measures (psychological flexibility, parental psychological inflexibility, and cognitive fusion). Secondary outcomes included parenting behavior, the quality of the parent-child interaction, parental competence, parental psychopathology, and the satisfaction with the treatment program. This is the first study we know of that showed statistically significant improvements on parental psychological flexibility, parenting behavior (except behavioral control), parent-child relations, parental competence, and parental internalizing psychopathology directly after treatment. At 6-month follow-up, significantly less conflicts, more parental competence, and less parental psychopathology was found. However, the improvements were not clinically significant, as calculated with the RCI. No control group was used in this uncontrolled pilot study but preliminary findings indicate that ACT parent counseling can help parents to increase psychological flexibility in order to choose more flexible and effective parenting behavior.
\end{abstract}

Keywords ACT $\cdot$ Parent counseling $\cdot$ Experiential avoidance $\cdot$ Psychological flexibility $\cdot$ Parenting behavior

\section{Highlights}

- This is the first pilot study that investigates Acceptance and Commitment Therapy (ACT) as parent counseling in parents with children with various psychopathology.

- Improvements on parental psychological flexibility, parenting behavior, parent-child relations, parental competence, and parental psychopathology were found.

- These preliminary findings indicate that ACT as parent counseling is a feasible and acceptable intervention for parents of children with various psychopathology.

It is generally accepted that ineffective parenting is associated with child psychopathology. For example,

Denise H. M. Bodden

D.Bodden@uu.nl

1 Child and Adolescent Studies, University of Utrecht, Utrecht, The Netherlands

2 Altrecht Child and Youth Psychiatry, Utrecht, The Netherlands several meta-analyses have shown associations between child psychopathology and more negative, avoidant, controlling, or overprotecting parenting (e.g. Yap \& Jorm, 2015; Pinquart, 2017). Besides, parents of children with psychopathology tend to experience more parental stress, guilt, and shame (Coyne \& Murrell, 2009). The causality and direction between child psychopathology and ineffective parenting is not clear yet but several theories have been developed to understand this association. 
According to the underlying theory of Acceptance and Commitment Therapy (ACT), parental psychological inflexibility plays an important role in explaining rigid and inflexible parenting. Psychological inflexibility can be described as a mismanagement of personal undesirable internal states (such as negative feelings, thoughts, and physical sensations) by using ineffective coping strategies like inhibition, suppression and/or avoidance which cause relief and stress reduction in the short term. In the long term, however, these ineffective strategies often rebound and cause paradoxical effects, so negative thoughts, emotions, dysregulation, and stress increase even more (Hayes \& Wilson, 1994). The two main processes of psychological inflexibility are Experiential avoidance and Cognitive fusion. Experiential Avoidance can be defined as attempts to control, modify, or avoid the frequency, intensity, or content of negative feelings, thoughts, or physical sensations. For example, parents might avoid conflicts with their child out of fear of being rejected by the child. Or parents use overprotection as a parenting behavior so the child will not get hurt. Parents tend to rely on experiential avoidance when they lack adequate resources to regulate their emotions in a different way, so experiential avoidance can be seen as an avoidant and ineffective emotion regulation strategy (Coyne et al., 2007). Cognitive fusion is the process of being trapped in negative thoughts such as "I fail as a parent" or "I am a bad parent." Parents tend to take these thoughts seriously which causes a lot of stress and feelings of incompetence (Coyne \& Wilson, 2004).

Several studies have been conducted, showing evidence for the theory that inefficient parenting is indeed related to parental psychological inflexibility (especially experiential avoidance). These studies have shown an association between parental inflexibility and the following parenting behaviors; less positive parenting (Brassell et al., 2016; Burke \& Moore, 2015), more negative parenting (Brassell et al., 2016), more inconsistent parenting (Berlin et al., 2006; Burke \& Moore, 2015; Shea \& Coyne, 2011), more harsh discipline (Brassell et al., 2016), more punitive parenting (Shea \& Coyne, 2011), poor monitoring (Berlin et al., 2006), laxness (Brassell et al., 2016; Burke \& Moore, 2015), overreactivity (Burke \& Moore, 2015), more control (Cheron et al., 2009), and overprotection (Fulton et al., 2014). Besides, more experiential avoidance is found to be related to inadequate communication of emotions (affective expression) (Cheron et al., 2009), more parental stress (Cheron et al., 2009; Moyer \& Sandoz, 2015; Shea \& Coyne, 2011), less parental sense of competence (Burke \& Moore, 2015), less control in the parenting role (Coyne \& Thompson, 2011) and more parental involvement (Berlin et al., 2006). These studies show the importance of psychological inflexibility (especially experiential avoidance) in parenting and parent-child interaction.
Besides the association between psychological inflexibility and ineffective parenting, also associations between parental experiential avoidance and child internalizing (Brassell et al., 2016; Cheron et al., 2009; Coyne \& Thompson, 2011; Fulton et al., 2014) and externalizing psychopathology (Brassell et al., 2016; Shea \& Coyne, 2011) have been found. These associations lead to research investigating whether parental psychological inflexibility leads to maladaptive or ineffective parenting behavior which in turn can lead to more child psychopathology (Dumas, 2005; Greco \& Eifert, 2004). In parents of adolescents, parental experiential avoidance indeed significantly predicted inconsistent discipline, poor monitoring and parental involvement, which in turn predicted adolescent behavior problems (Berlin et al., 2006). It was also suggested that psychological inflexibility prevents parents from changing their parenting behavior (Coyne \& Wilson, 2004; Murrell et al., 2008; Tiwari et al., 2008), reduces options for positive experiences and limits parents in their response options (Williams et al., 2012). These studies make it plausible that changing psychological inflexibility into psychological flexibility could be the answer to ineffective parenting.

Increasing one's Psychological flexibility is the main goal of Acceptance and Commitment therapy (ACT). ACT is a treatment that focusses on acceptance of unpleasant emotions, defusion from difficult thoughts, clarification of values and goals, and enhancement of reaching those values and goals. Psychological flexibility can be defined as an individual's acceptance of negative feelings, thoughts, and physical sensations and the ability to choose an adaptive (and more effective) response. Psychological flexibility is the opposite of psychological inflexibility and the main two process involved are Acceptance and Cognitive defusion. Acceptance is the willingness to accept negative feelings, thoughts, and physical sensations fully without struggle or control, given one's values. For example, parents learn to cope with their child being angry at them when they set clear boundaries. Cognitive defusion is the process of entangling one's self from thoughts in such a way that thoughts can be seen as thoughts and not as facts. For example, parents learn to realize that the thought of failing of falling short is just a thought and not a fact. Changing parents from being psychological inflexible to being more psychological flexible could be the key process that is necessary to ultimately change ineffective parent behavior.

Usually parent training is focused on the psychopathology of the child and often involves improving parenting behavior directly to address the child's behavior. However, the psychological needs of parents themselves are often ignored (Blackledge \& Hayes, 2006). Therapists often fail to acknowledge these within-parent factors and focus on parenting behavior. But because of these cognitive and emotional challenges (psychological inflexibility), changes in parenting are difficult to achieve (Coyne \& Wilson, 2004; 
Murrell et al., 2008; Tiwari et al., 2008). That is why we developed a parent intervention based on ACT which is directed at parental psychological inflexibility. In this intervention, ACT as parent counseling, parents learn to decrease experiential avoidance and cognitive defusion in order to increase more flexible parenting behaviors.

Previous research on ACT as a parent intervention has shown promising results. A two day ACT intervention for parents of children $(n=20)$ diagnosed with autism, showed improvements on depression, psychological distress, general health, cognitive fusion, and experiential avoidance at 3 month follow-up (Blackledge \& Hayes, 2006). Another study on parents of children with autism $(n=13)$ showed a decrease in distress after 8 weeks ACT (Kowalkowski, 2012). Parents of children with developmental disabilities $(n=27)$ showed improvements in mental health after ACT workshops, but no changes on ACT measures were found (Tani et al., 2013). A study comparing Stepping Stones Triple P (SSTP) $(n=22)$ with SSTP + ACT $(n=23)$ and waitlist $(n=22)$ for parents of children with cerebral palsy showed a decrease in child behavioral problems and parental over reactivity and verbosity in the SSTP + ACT condition. No differences between the SSTP and SSTP + ACT condition were found (Whittingham et al., 2014). Another study investigating the effects of a 10 week group SSTP + ACT with parents of children with acquired brain injury $(n=59)$ showed that a combination of SSTP + ACT was more effective in decreasing child problem behavior, parental stress, overreactive and lax parenting and in increasing parent psychological flexibility, parent confidence in managing behaviors and family adjustment compared to solely parent training (Brown et al., 2014, 2015). Also case studies have shown some evidence for the applicability for ACT as a parent intervention in combination with Parent-Child Interaction Therapy (PCIT; Ascanio \& Garcia, 2018; Coyne \& Wilson, 2004) and Behavioral Parent Training ( $n=3$, Cohrs, 2012). So there has been some evidence to support the applicability and preliminary effects of ACT as a parent intervention. However research is scarce and the methodological quality is often poor (small sample sizes), so more research is warranted (Coyne et al., 2011; Raftery-Helmer et al., 2016). This study is the first uncontrolled pilot study that investigates ACT as parent counseling in a moderately sized sample of parents $(n=101)$ who have children with various psychopathology. Also, outcomes covering a broad range of parenting behaviors as well as the parent-child relationship were used.

In this uncontrolled pilot study, ACT is provided as a parent counseling therapy in order to increase psychological flexibility which in turn helps parents with their parenting behavior, parent-child relationship problems, and feelings of incompetence. The ACT parent counseling consists of nine grouptherapy sessions with six to eight parents. Our hypotheses were that after the ACT parent counseling parents report improvements of (1) psychological flexibility, parental psychological flexibility, and cognitive fusion, (2) parenting behavior (more consistency, responsiveness, and less psychological and behavioral control), (3) the quality of the parent-child relationship (better communication and less conflicts), (4) parental sense of competence, and (5) Parental psychopathology. Besides, (6) treatment satisfaction was measured.

\section{Method}

\section{Participants}

In total, 114 parents of children with psychiatric problems have participated in the ACT parent counseling. The child's age was between three and 18 years old $(m=14.3, s d=$ 3.1 ), the majority was 13 years an above $(71.7 \%)$ and is referred to an outpatient mental health care facility (Altrecht) because of psychiatric problems. All children had a clinical diagnosis namely Depressive Disorder (23.8\%), Pervasive Developmental Disorder (19.8\%), Attention Deficit Hyperactivity Disorder (12.9\%), Anxiety disorder (10.9\%), Oppositional Defiant Disorder (7.9\%), Conduct Disorder (3\%), Adjustment Disorder (2\%), ObsessiveCompulsive Disorder (2\%), Autism (1\%), Eating Disorder (1\%), Attachment Disorder (1\%), Psychotic Disorder (1\%), and Somatoform Disorder (1\%). We could not retrieve the diagnostic status of $12 \%$ of the children. Parents could participate if they experienced problems related to stimulating the development of the child because of (1) own psychopathology, (2) difficulties with reflecting on or adjusting their parenting behavior, (3) being stuck in ineffective parenting behavior, (4) structural problems in their parent-child interactions, (5) feelings of incompetence regarding their parenting, or (6) having problems maintaining their own parenting ideas. Exclusion criteria were psychiatric problems with the risk of dissociation, not fluent in Dutch, or an IQ below 80 (see procedure).

Nine parents $(8 \%)$ were included but eventually did not start the intervention and the measurements because of (being overworked $(n=3)$, own psychopathology $(n=2)$, own physical problems $(n=1)$, crisis of the child $(n=3))$. Four parents $(4 \%)$ refused to fill out the questionnaires but did follow the intervention. In total 101 of the parents filled out the pretreatment assessment. Only 6 (5\%) participants dropped-out of treatment and did not fill out posttreatment measurements because two parents could not get a day off work, a parent couple had a family member that died $(n=2)$, one parent had difficulties understanding the assignments and one parent did not have a connection with the other group members. The reaming 95 parents completed all nine sessions. After the intervention, 20 parents did not fill out the posttreatment measurements leaving $75(74 \%)$ parents that completed the post-treatment assessment. At follow-up it was 
even harder to motivate and trace parents and another 32 parents were considered follow-up measurement drop outs. Eventually, 43 (43\%) parents completed the 6-month followup. See participants flow chart, Fig. 1.

The mean age of the included parents at pretreatment was 47.1 years $(s d=6.8$, range $=30-66)$ and the majority was female $(72 \%)$. No diagnostic status was obtained for the parents. Most parents $(n=98,97 \%)$ had the Dutch Nationality, three parents had a different nationality namely French $(1 \%)$, American (1\%), and Surinamese (1\%). Parent's highest educational level was middle level (37\%) and high level (53\%). Most parents had a paid job (78\%), 22\% had no paid job (of which $50 \%$ housewife, $23 \%$ disabled, $18 \%$ unemployed, and $9 \%$ retired). Eighty percent of the parents were living together with a partner (married $64 \%$, living together $14 \%$, and living together after divorce $2 \%$ ) and $20 \%$ was in a single parent family ( $14 \%$ divorced and alone and $6 \%$ alone or widow). The parents attended the intervention for one child who was in treatment for psychopathology. The mean number of children was $2.1(s d=0.75$, range $=1-4)$. Parents who completed the assessments at posttreatment were comparable to parents who did not fill out the subsequent assessment at posttreatment $(n=26)$ in terms of gender $\left(\chi^{2}(1, n=101)=0.83, p>0.05\right)$, educational level $\left(\chi^{2}(4, n=101)=0.91, p>0.05\right)$, age $F(1$, $99)=3.37, p>0.05$, initial level of internal psychopathology $F(1,99)=0.34, p>0.05$, initial level of external psychopathology $F(1,99)=3.57, p>0.05$, and initial level of experiential avoidance $F(1,99)=0.75, p>0.05$.

In this research, a repeated measures design was used. These data provide a first indication of effectiveness. No

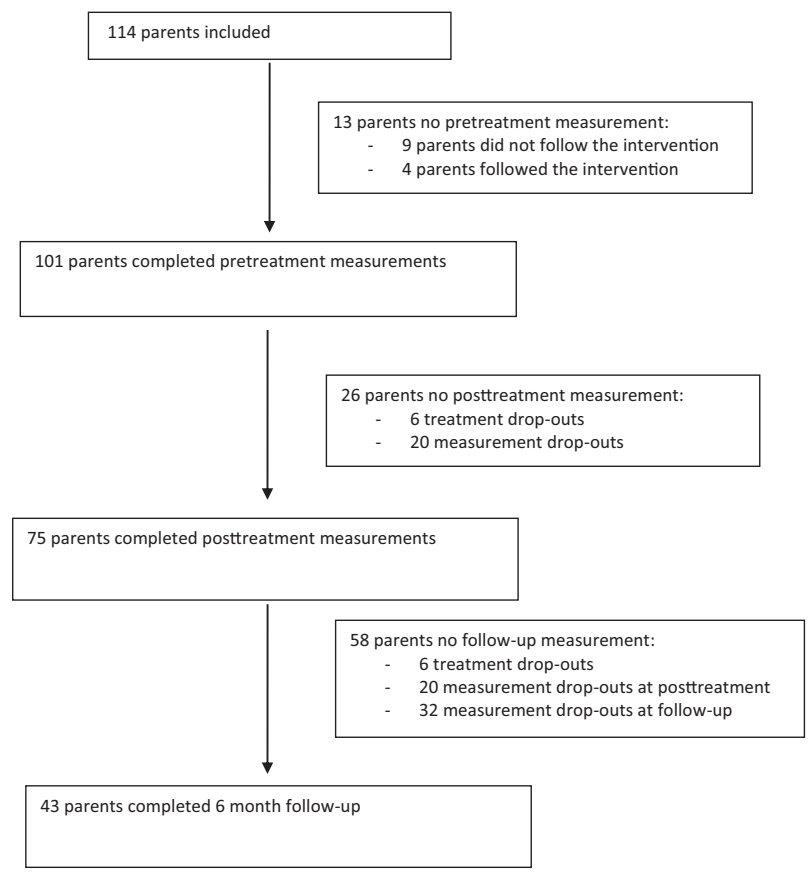

Fig. 1 Participants flow chart control group was included because of lack of an appropriate care as usual control group. In the Netherlands, there are no guidelines concerning parent counseling when the child is treated for psychopathology. Sometimes parents are not involved at all, sometimes they receive some sessions psychoeducation about the child's psychopathology and other times they receive multiple sessions including parenting advice. Since care as usual is very diverse in content and in frequency it is difficult to compare with.

The lack of a control group poses a threat to the internal validity of the study, limiting the conclusions drawn from this uncontrolled pilot study in that the observed changes could also be caused by other factors besides the treatment. However this study is the first pilot study investigating the potential effects of ACT as parent counseling giving us an indication whether we should proceed with this type treatment. The design and results can also enhance the feasibility, acceptability, and quality of follow-up studies in this field. Three assessments were conducted: prior to the ACT counseling (pretreatment), directly after the ACT counseling (post-treatment), and 6 months after ACT counseling (6month follow-up). Parents' self-reports were used.

\section{Procedure}

Parents, whose child was in treatment for psychopathology, were asked by their child's psychologist to participate in ACT parent counseling. First they were screened for the inclusion and exclusion criteria and their willingness to participate in ACT by means of an introductory meeting with the ACT therapists (psychologists). Parents could participate if they experienced problems related to stimulating the development of the child because of (1) own psychopathology (e.g. anxiety, depression, etc.), (2) difficulties with reflecting on or adjusting their parenting behavior, (3) being stuck in in-effective parenting behavior (e.g. overprotection, permissive, critical, too much control), (4) structural problems in their parent-child interactions (e.g. conflict, attachment problems), (5) feelings of incompetence regarding their parenting, or (6) having problems maintaining their own parenting ideas. During the meeting, these problems were discussed and if parents met one of these inclusion criteria, they were invited to participate. Exclusion criteria were psychiatric problems with the risk of dissociation, not fluent in Dutch, or an IQ below 80. The latter two were observed during the meeting. The risk of dissociation was checked by asking if the parent had psychotic of delusion problems. In this meeting, parents also received a brochure and verbal information about ACT and the study and were asked to participate. Prior to the treatment (pre-treatment) parents filled out questionnaires and signed the informed consent. Then parents followed nine ACT parent counseling meetings. In the last session, parents 
filled out the questionnaires after the treatment (post-treatment). Six months after treatment (6-month follow-up) parents received the follow-up questionnaires by mail.

\section{Measures}

Psychological flexibility will be measured using the Acceptance and Action Questionnaire (AAQ-II; Bond et al., 2011; Dutch version; Jacobs et al., 2008). The AAQ-II is a ten item self-report questionnaire to measure emotional avoidance and emotion-fused inaction on a seven point Likert scale ranging from (1) never true to (7) always true. A high score indicates high psychological flexibility (more acceptance and less experiential avoidance). The Cronbach's alpha in our study was 0.90 at pretreatment.

The Dutch Parental Acceptance and Action Questionnaire (PAAQ; Cheron et al., 2009; Dutch version; Bodden et al., 2012) was used to measure the level of experiential avoidance parents have towards the problem events of their children, so their psychological inflexibility. The PAAQ consists of 15 items and parents have to indicate the truth of each item as it applies to them on a seven point Likert scale ranging from (1) never true to (7) always true. A high score reflects more parental Experiential Avoidance in childrearing situations, in other words the way parent's try to avoid or control situations in which their child experiences negative emotions and the parent's inability to effectively manage their own reaction to their child's affect. The internal consistency was found to be moderate (0.640.65; Cheron et al., 2009). The Cronbach's alpha in our study was 0.76 at pretreatment.

Cognitive fusion was measured using the Cognitive Fusion Questionnaire (CFQ; Gillanders et al., 2014; Dutch version; Batink \& De Mey, 2011). The CFQ contains of 13 items which are rated on a seven point Likert scale ranging from (1) never true to (7) always true. The higher the score, the more fused one is with one's thoughts. The Cronbach's alpha in our study was 0.90 at pretreatment.

Different subscales of various parental self-report questionnaires were used to measure important parenting behavior. Eight items from the Parenting Dimensions Inventory (Slater \& Power, 1987; Dutch version Gerrits et al., 1997) were used to measure consistency, the degree to which the parent shows predictable penalty behavior. Items were rated on a six point Likert scale ranging from (1) totally disagree to (6) totally agree. The Cronbach's alpha was 0.83. Eight items from The Nijmegen Rearing Questionnaire (NOV; Gerris et al., 1993) were used to measure parental responsiveness, the degree to which the parent is responsive to the needs, signals, and condition of the child. Items were rated on a scale from (1) totally disagree to (6) totally agree. The Cronbach's alpha was 0.89. Psychological control, the degree in which the parent tries to control the child in an intrusive way is measured using the Psychological Control Scale (PCS; Barber, 1996). The eight items were rated on a six point Likert scale from (1) totally disagree to (6) totally agree. The Cronbach's alpha was 0.73 . Six items from the Parental Monitoring Scale (Deković et al., 2003) were used to measure behavioral control; the degree to which parents monitor their children. The items were rated on a four point scale ranging from (1) (I know nothing about it) to 4 (I know all about it). The Cronbach's alpha was 0.83 .

The subscales communication and the degree of conflicts were used to measure the quality of the parent-child relationship. The degree of openness and the extent of problems that prevent or hinder communication between parent and child (communication) was measured with nine items of the Parent Child Communication Scale (PCS; Barnes \& Olson, 1985). Items were rated on a six point Likert scale from (1) totally not applicable to (6) very applicable. A high total score reflects a good parent-child communication. Cronbach's alpha was 0.75 . The degree of conflicts (quarrels, irritations, and antagonism in the child-parent relationship) was measured using the six-item Network of relationship inventory (NRI; Furman \& Buhrmester, 1985). Parents can indicate whether they have conflicts (1) few tot none to (5) very much (more is not possible) conflicts with a high score reflecting more conflicts. The alpha was 0.93 .

Parental competence was measured using the 16-item Parental Sense of Competence scale (PSOC), which measures satisfaction (reflecting parenting frustration, anxiety, and motivation, liking the parenting role) and efficacy (reflecting competence, problem solving ability, and capability in the parenting role). Items are measured on a six point Likert scale ranging from (1) totally agree to (6) totally disagree (Johnston \& Mash, 1989). Cronbach's alpha was 0.81 .

The commonly used Adult Self-Report (ASR; Achenbach \& Rescorla, 2003) was used to measure parental psychopathology. The ASR contains 69 items summing up to two global syndrome scales namely Internalizing problems and Externalizing problems. A higher score indicating more psychopathology. Cronbach's alpha was 0.90 for internalizing problems and 0.82 for externalizing problems.

We also asked parents whether they felt like the ACT parent counseling had helped them using yes-no questions. Satisfaction with treatment was measured using the ten-item Satisfaction with Program Scale (SPS; Tolan et al., 2002).

\section{ACT Parent Counseling}

ACT parent counseling consists of ten group sessions (including one introduction meeting, eight sessions and one follow-up meeting) which last about 2 to 3 months. The content of the intervention is described in a therapist manual combined with workbook "ACTive parenting" (Matthijssen, 2012) for parents. The first phase of the intervention 
is focused on values in parenting and the needs of the child. Parents learn to focus on behavior that they think is helpful for development of their child. After that parents try to identify automatic responses, caused by fusion and experiential avoidance. The second phase is directed at acquiring competences related to mindfulness, acceptance, and commitment with chosen values in parenting. In each group, six to eight parents participated (also parent couples participated in the same group). Regular exercises are; mindfulness exercise, a theme directly connected to the clinical experience of parents and experiential exercises. The manual of this intervention is inspired by a group protocol by Bockstaele and Pascal-Claes (2010), "The Joy of Parenting" (Coyne \& Murrell, 2009) and "Parenting Your Anxious Child with Mindfulness and Acceptance: A Powerful New Approach to Overcoming Fear, Panic, and Worry Using Acceptance and Commitment Therapy" (McCurry, 2009).

\section{Data Analyses}

Missing data on the item level were imputed using the missing value analysis of SPSS. No more than $8 \%$ of the items were missing per questionnaire. Mean scores were used. To evaluate the effect at post-treatment and follow-up, repeated t-tests were conducted. The effect size was computed to indicate the degree of difference between the two measurements. For each questionnaire the effect size (Cohen's d) from pre- to post-treatment was defined as (Mpre-Mpost)/SDpooled, where Dpooled = [(SDpre2 + SDpost2)/2]. For follow-up pre and follow-up measurements were compared. Given the large amount of tests, we used Bonferroni correction to reduce the Type I error. We adjusted the $\mathrm{p}$ value by dividing the critical $\mathrm{P}$ value $(\alpha=0.05)$ by the number of comparisons made. Effect sizes were interpreted as large $(>0.50)$, medium $(0.30-0.50)$, and small (0.10-0.30) (Field, 2009).

Additionally, to give an indication of clinical significance, we calculated the Reliable Change Index (RCI; Jacobson \& Truax, 1991) by dividing the difference between the pretreatment and posttreatment scores by the standard error of the difference between the two scores. If the RCI is $>1.96$, then the difference is reliable. The RCI is one of the most commonly used and cited methods for examining clinical significance. Two reviews also concluded that the RCI can be used to indicate whether or not the client improved (Ogles et al., 2001; Wise, 2004).

\section{Results}

\section{Effects at Post-treatment}

The completer analyses (with Bonferroni correction) showed that ACT outcomes improved significantly. Parents reported significantly less parental psychological inflexibility (less experiential avoidance) and less cognitive fusion after receiving ACT parent counseling. Effect sizes were small to large.

Also secondary outcomes improved. Parents reported their parenting to be more consistent (medium effect size) and more responsive (small effect size). No significant differences (using Bonferroni correction) between pre and post-treatment were found on psychological control, and behavioral control. The quality of parent-child relations also increased significantly; parents reported an improvement on communication and less conflicts (small effect sizes). Besides, parents reported feeling significantly more competent regarding their parenting (large effect size). Lastly, parents own internal psychopathology significantly decreased after ACT parent counseling (small effect size). Parents own external psychopathology did not decrease significantly after the Bonferroni correction, see Table 1. Intent-to-treat analyses showed exactly the same significant (all $t$ 's between -5.54 and $4.67, p$ 's $<0.019$ ) and nonsignificant findings (all $t$ 's between -1.10 and 1.47, $p$ 's $>0.05)$.

\section{Effects at 6-month Follow-up}

At 6-month follow-up, improvements on ACT processes were still present in the completer analyses. Parents reported more psychological flexibility, less parental psychological inflexibility, and less cognitive fusion but the significance level did not reach the Bonferroni adjusted $\mathrm{p}$ value. Effect sizes were medium to large. Regarding parenting, parents reported more consistent (medium effect size) and responsive (small effect size) parenting and less psychological control (medium effect size). However, again the $p$ values did not reach the Bonferroni adjusted $p$ value. No significant difference between pretreatment and 6-month follow-up was found on behavioral control and communication. Conflicts decreased significantly after the Bonferroni correction (medium effect size). Also, significantly more parental competence was reported by parents (large effect size). Large effect sizes were found regarding parents own psychopathology; both internalizing and externalizing psychopathology significantly decreased after Bonferroni correction, see Table 2.

\section{Reliable Change Index}

As an indication of clinical significance, we calculated the Reliable Change Index (RCI; Jacobson \& Truax, 1991). Although we did find significant changes, none of them had a RCI higher than 1.96 suggesting no reliable difference in other words no clinical significant improvements were found. The RCI's ranged from 0.19 (parental internalizing 
Table 1 Means and standard deviations on parent reports at pretreatment and post-treatment $(n=75)$

Table 2 Means and standard deviations on parent reports at pretreatment and 6-month follow-up $(n=43)$

\begin{tabular}{|c|c|c|c|c|c|}
\hline & $\begin{array}{l}\text { Pretreatment } \\
M(S D)\end{array}$ & $\begin{array}{l}\text { Post-treatment } \\
M(S D)\end{array}$ & $T$ & $E S$ & $R C I$ \\
\hline \multicolumn{6}{|l|}{ ACT measures } \\
\hline Psychological flexibility & $4.89(1.02)$ & $5.12(0.90)$ & -2.52 & -0.24 & 0.89 \\
\hline Parental psy. inflexibility & $3.76(0.58)$ & $3.35(0.45)$ & $5.18 * *$ & 0.79 & 0.79 \\
\hline Cognitive fusion & $3.82(0.82)$ & $3.34(0.71)$ & $4.92 * *$ & 0.63 & 0.72 \\
\hline \multicolumn{6}{|l|}{ Parenting } \\
\hline Consistency & $3.71(0.94)$ & $4.00(0.83)$ & $-4.07 * *$ & -0.33 & 1.07 \\
\hline Responsiveness & $4.27(0.82)$ & $4.49(0.79)$ & $-3.86 * *$ & -0.27 & 0.75 \\
\hline Psychological control & $2.44(0.68)$ & $2.27(0.69)$ & 2.41 & 0.25 & 0.98 \\
\hline Behavioral control & $3.09(0.43)$ & $3.07(0.44)$ & 0.68 & 0.05 & 0.49 \\
\hline \multicolumn{6}{|l|}{ Parent-child relationship } \\
\hline Communication & $3.82(0.87)$ & $4.01(0.88)$ & $-3.32 * *$ & -0.22 & 1.21 \\
\hline Conflicts & $2.13(0.68)$ & $1.95(0.58)$ & $3.88 * *$ & 0.28 & 0.50 \\
\hline \multicolumn{6}{|l|}{ Parental competence } \\
\hline Parental competence & $3.67(0.59)$ & $3.98(0.65)$ & $-5.84 * *$ & -0.50 & 0.71 \\
\hline \multicolumn{6}{|l|}{ Parental psychopathology } \\
\hline Intern. psychopathology & $0.38(0.22)$ & $0.32(0.21)$ & $3.07 * *$ & 0.28 & 0.19 \\
\hline Extern. psychopathology & $0.25(0.17)$ & $0.20(0.16)$ & 2.74 & 0.30 & 0.20 \\
\hline
\end{tabular}

$* * p<0.003$ (Bonferroni correction)

\begin{tabular}{|c|c|c|c|c|c|}
\hline & $\begin{array}{l}\text { Pretreatment } \\
M(S D)\end{array}$ & $\begin{array}{l}\text { Follow-up } \\
M(S D)\end{array}$ & $T$ & $E S$ & $R C I$ \\
\hline \multicolumn{6}{|l|}{ ACT measures } \\
\hline Psychological flexibility & $4.97(0.95)$ & $5.30(0.92)$ & -2.92 & -0.35 & 0.83 \\
\hline Parental psy. inflexibility & $3.71(0.57)$ & $3.21(0.44)$ & 3.17 & 0.98 & 0.77 \\
\hline Cognitive fusion & $3.66(0.84)$ & $3.31(0.77)$ & 2.44 & 0.43 & 1.14 \\
\hline \multicolumn{6}{|l|}{ Parenting } \\
\hline Consistency & $3.72(0.98)$ & $4.00(0.91)$ & -3.04 & -0.30 & 1.12 \\
\hline Responsiveness & $4.27(0.86)$ & $4.50(0.89)$ & -2.95 & -0.26 & 0.79 \\
\hline Psychological control & $2.40(0.66)$ & $2.18(0.74)$ & 2.29 & 0.31 & 0.95 \\
\hline Behavioral control & $3.13(0.42)$ & $3.03(0.47)$ & 1.42 & 0.22 & 0.48 \\
\hline \multicolumn{6}{|l|}{ Parent-child relationship } \\
\hline Communication & $3.79(1.03)$ & $4.05(1.02)$ & -2.78 & -0.25 & 1.43 \\
\hline Conflicts & $2.24(0.76)$ & $1.97(0.66)$ & $3.29 * *$ & 0.38 & 0.56 \\
\hline \multicolumn{6}{|l|}{ Parental competence } \\
\hline Parental competence & $3.63(0.64)$ & $3.97(0.72)$ & $-4.29 * *$ & -0.50 & 0.77 \\
\hline \multicolumn{6}{|l|}{ Parental psychopathology } \\
\hline Intern. psychopathology & $0.37(0.22)$ & $0.27(0.18)$ & $4.28 * *$ & 0.50 & 0.19 \\
\hline Extern. psychopathology & $0.24(0.15)$ & $0.16(0.16)$ & $4.01 * *$ & 0.52 & 0.18 \\
\hline
\end{tabular}

$* * p<0.003$ (Bonferroni correction) psychopathology) to 1.21 (communication) at posttreatment and from 0.18 (parental externalizing psychopathology) to 1.43 (communication), see Tables 1 and 2 . These results indicate that the magnitude of change of ACT parent counseling was not clinically significant.

\section{Satisfaction with Treatment}

Parental Satisfaction with the therapist $(m=4.90, s d=$ 0.82 , range $1-6)$ and treatment $(m=5.53, s d=0.87$, range 1-6) was high. We also asked parents whether they 
felt like the ACT parent counseling had helped them (yes or no). Almost all parents $97 \%$ indicated they felt as though the ACT parent counseling helped them as a person, $91 \%$ felt the intervention helped them as a parent, $80 \%$ indicated that the parent-child relationship improved, $75 \%$ indicated that the child benefitted, and $60 \%$ indicated that their relationship with their significant other had improved because of the treatment.

\section{Discussion}

The aim of this uncontrolled pilot study was to investigate whether ACT as parent counseling affects parents of children in treatment for psychopathology. Results show that ACT outcomes (parental psychological flexibility and cognitive fusion), parenting (consistency and responsiveness), the parent-child relations (communication and conflicts), and parental competence improved and parental internalizing psychopathology significantly decreased directly after treatment. At 6-month follow-up, significant improvements were found on conflicts, parental competence and parental internalizing and externalizing psychopathology. However, no clinical significance was found.

ACT is focused on changing the degree of psychological flexibility and less so on reducing psychopathology (in contrast to for example CBT). In our research, the mechanism through which ACT is supposed to work parental psychological flexibility - indeed improved. More specifically, parents reported more parental psychological flexibility (less experiential avoidance) and less cognitive fusion after receiving ACT parent counseling. This is in line with previous research showing improvements on cognitive fusion and experiential avoidance after a 2 day ACT workshop (Blackledge \& Hayes, 2006) and psychological flexibility after a 10 week group SSTP + ACT training (Brown et al., 2014, 2015). Psychological flexibility could function as a outcome measure but it could as well be a mediating mechanism. Due to the lack of measurements during treatment, we could not measure this mediator effect. In our study, we did find improvements on parenting reported by parents. These changes could be caused by the fact that parents were more psychological flexible in parenting after ACT parent counseling. This was also suggested by previous research in which the allegedly theoretical working mechanism of ACT (psychological flexibility) is indeed pinpointed as the process through which ACT works in adult studies (Hayes et al., 2006). But also in a study investigating SSTP + ACT in a parent group format, reductions in experiential avoidance mediated the treatment effect on reducing maladaptive parenting and parental stress (Brown et al., 2015).

Statistically significant improvements on parenting were also found after ACT parent counseling. More specifically, parents reported being more consistent and more responsive in their parenting. This is the first pilot study that has investigated the effects on various parenting behavior after an ACT parent counseling. Previous studies only focused on some aspects of parenting for example overreactive and lax parenting (Brown et al., 2014) or parental over reactivity and verbosity (Whittingham et al., 2014). As already mentioned, changes in parenting behavior could be an indirect effect of the change in experiential avoidance. As in the Brown et al. (2015) study reductions in experiential avoidance could have mediated the treatment effect on reducing maladaptive parenting. In contract to expectations, no changes were found on psychological control and behavioral control directly after the intervention.

Besides parenting, the parent-child relations also improved significantly according to parents. Communication between parents and their child improved and they had less conflicts after ACT parent counseling. No prior study investigating ACT as parent counseling has investigated the parent-child relationship so no comparisons can be made.

Parental sense of competence also improved directly after the ACT parent course and at 6 month follow-up. A previous study on SSTP + ACT also reported more parent confidence in managing behaviors (Brown et al., 2014, 2015). Moreover, several studies have shown that parental stress reduces after ACT parent interventions (Blackledge \& Hayes, 2006; Brown et al., 2014; Kowalkowski, 2012). We did not measure parental stress in general but we assume that the increase of parental competence could have led to a decrease in parental stress as well.

Parents' self-reported psychopathology also significantly decreased after ACT parent counseling and both internal and external psychopathology significantly decreased at 6 month follow-up according to parental self-report. This finding is consistent with meta-analyses showing that ACT is effective in reducing depression, anxiety, addiction, somatic health problems in adults (A-tjak et al., 2015). Also studies on parent interventions show decreases on depression (Blackledge \& Hayes, 2006) and mental health (Tani et al., 2013) after ACT workshops. Reductions in parental psychopathology might also be an indirect effect caused by improved psychological flexibility. Several studies have shown the association between experiential avoidance and psychological wellbeing in adults (weighted effect size $=$ 0.42; Hayes et al., 2006) but also in parental experiential avoidance and parental psychopathology (Brassell et al., 2016; Cheron et al., 2009; Coyne \& Thompson, 2011; Moyer \& Sandoz, 2015; Shea \& Coyne, 2011). 
Although we did find statistical significant changes, none of them had a RCI higher than 1.96 suggesting that none of the parents clinically improved. The RCI method is able to take into account different practice effects and other sources of variance when determining treatment change. We measured clinical significance at group level, ruling out clinical significance at individual level. Measuring at group level, also makes the RCI a very conservative and strict measure. These findings indicate the need to replicate these findings in a larger sample size and compare them to a control group.

Treatment satisfaction was high and parents reported that ACT parent counseling was very helpful. This suggests that parents like the intervention and that they feel it's beneficial. Also, only $5 \%$ of the parents discontinued treatment, so treatment adherence is high. Both findings indicate a high level of acceptability of ACT as parent counseling.

\section{Limitations}

Since this was a trial conducted in clinical practice without any financial aids, we could not conduct a randomized controlled trial. A randomized controlled trial is the only design that can reliably establish effectiveness. Also a control group was difficult to establish since we could not match the investigated intervention with another available intervention in terms of content, goal, and target group. In absence of a control group, we cannot conclude that the observed changes in outcome can be attributed to the ACT parent counseling intervention alone. Possible confounding factors such as the treatment of the child might have also caused the change in outcomes. Therefore, it is not possible to conclude that ACT as parent counseling is effective. Besides, there was a high measurement attrition rate at posttreatment (26\%). However a large proportion of these participants (77\%) did not fill out the measurements at posttreatment and only a few parents drop out of treatment (23\%). In total 6\% (six parents) did not continue ACT parent counseling suggesting high treatment adherence. At follow-up, even more parents did not complete the assessment (57\%). Lastly, we relied solely on parent report. Parents might have responded in a socially desirable manner. We did not include child report or observations which might have given a different view on parenting and the parent-child relationship. Combining parent and child report would increase the reliability of the findings. The lack of control group, the high attrition rate and the use parent-report only pose a serious threat to the validity and reliability of this study, so results of this study should be interpreted with caution.

Notwithstanding these limitations, this uncontrolled pilot study is the first to investigate ACT as a parent counseling in parents with children with various psychopathology. Also, a moderately sized sample size and multiple outcome measures were used. In this pilot study, some indications for the potential effects of ACT as parent counseling have been found. In the future, we hope to conduct a control group design study (preferably a RCT), which will shed more light on the effectiveness of ACT parent counseling.

\section{Clinical Implications}

Usually parent counseling is focused on the psychopathology of the child and ignores the psychological needs of parent. Parents have negative thoughts and emotions about the child's psychopathology, their parenting and their relation with the child. In order to change parenting behavior and the parent-child relations, parental psychological flexibility should be increased first. However, in clinical practice, this step is often left out and parents receive parent training without working on their inner challenges or psychological inflexibility first. This study shows that ACT parent counseling increases psychological flexibility, parenting, parent-child relations also changes parenting, the parent-child relations, parental sense of competence and parental psychopathology. Although the changes were not clinical significant, parents were very satisfied with the intervention and few parents dropped-out of treatment. This indicates that ACT as parent counseling is a feasible and acceptable intervention for parents of children with various psychopathology.

Author Contributions All authors contributed to the study conception and design. All authors read and approved the final manuscript.

\section{Compliance with Ethical Standards}

Conflict of Interest The authors declare no competing interests.

Ethical Approval All procedures performed in studies involving human participants were in accordance with the ethical standards of the institutional research committee (Commissie Wetenschappelijk Onderzoek Altrecht, April 2011) and with the 1964 Helsinki declaration and its later amendments or comparable ethical standards.

Informed Consent Informed consent was obtained from all individual participants included in the study.

Publisher's note Springer Nature remains neutral with regard to jurisdictional claims in published maps and institutional affiliations.

Open Access This article is licensed under a Creative Commons Attribution 4.0 International License, which permits use, sharing, adaptation, distribution and reproduction in any medium or format, as long as you give appropriate credit to the original author(s) and the source, provide a link to the Creative Commons license, and indicate if changes were made. The images or other third party material in this article are included in the article's Creative Commons license, unless indicated otherwise in a credit line to the material. If material is not included in the article's Creative Commons license and your intended use is not permitted by statutory regulation or exceeds the permitted use, you will need to obtain permission directly from the copyright holder. To view a copy of this license, visit http://creativecommons. org/licenses/by/4.0/. 


\section{References}

Achenbach, T. M., \& Rescorla, L. A. (2003). Manual for the ASEBA Adult Forms and Profiles. Burlington, VT: University of Vermont, Research Center for Children, Youth, and Families.

Ascanio, L., \& García, R. F. (2018). Combinando la terapia de aceptación y compromiso con la terapia de interacción padres-hijos en un niño con graves problemas de conducta. Revista de Psicología Clínica con Niños y Adolescentes, 5(1), 57-62.

A-tjak, J. G., Davis, M. L., Morina, N., Powers, M. B., Smits, J. A., \& Emmelkamp, P. M. (2015). A meta-analysis of the efficacy of Acceptance and Commitment Therapy for clinically relevant mental and physical health problems. Psychotherapy and Psychosomatics, 84(1), 30-36. https://doi.org/10.1159/000365764.

Batink, T. \& De Mey, H. R. A. (2011). De Nederlandstalige Cognitive Fusion Questionnaire (CFQ13). Retrieved from http://contextua lpsychology.org/cognitive_fusion_questionnaire_dutch_translation.

Barber, B. K. (1996). Parental psychological control: Revisiting a neglected construct. Child Development, 67, 3296-3319. https:// doi.org/10.1111/j.1467-8624.1996.tb01915.x.

Barnes, H. L., \& Olson, D. H. (1985). Parent-adolescent communication and the circumplex model. Child Development, 56, 438-447. https://doi.org/10.2307/1129732.

Berlin, K. S., Sato, A., \& Jastrowski, K. (2006). Effects of experiential avoidance on parenting practices and adolescent outcomes [Paper presentation]. Chicago: Association for Behavioral and Cognitive Therapies.

Blackledge, J. T., \& Hayes, S. C. (2006). Using acceptance and commitment training in the support of parents of children diagnosed with autism. Child \& Family Behavior Therapy, 28, 1-18. https://doi.org/10.1300/J019v28n01_01.

Bockstaele, M., \& Pascal-Claes, F. (2010). ACT groepsprotocol. CGG Waas \& Dender and Sint-Niklaas.

Bodden, D., Matthijssen, D., Stikkelbroek, Y. \& Hale, B. (2012). The Dutch translation and adaptation of the Parental Acceptance and Action Questionnaire (PAAQ). Unpublished Manuscript, University of Utrecht.

Burke, K., \& Moore, S. (2015). Development of the parental psychological flexibility questionnaire. Child Psychiatry \& Human Development, 46(4), 548-557. https://doi.org/10.1007/s10578-014-0495-x.

Bond, F. W., Hayes, S. C., Baer, R. A., Carpenter, K. M., Orcutt, H. K., Waltz, T., \& Zettle, R. D. (2011). Preliminary psychometric properties of the Acceptance and Action Questionnaire -II: A revised measure of psychological flexibility and experiential avoidance. Behavior Therapy, 42(4), 676-688. https://doi.org/10.1016/j.beth.2011.03.007.

Brassell, A. A., Rosenberg, E., Parent, J., Rough, J. N., Fondacaro, K., \& Seehuus, M. (2016). Parent's psychological flexibility: Associations with parenting and child psychosocial well-being. Journal of Contextual Behavioral Science, 5(2), 111-120. https://doi. org/10.1016/j.jcbs.2016.03.001.

Brown, F. L., Whittingham, K., Boyd, R. N., McKinlay, L., \& Sofronoff, K. (2015). Does Stepping Stones Triple P plus Acceptance and Commitment therapy improve parent, couple, and family adjustment following paediatric acquired brain injury? A randomised controlled trial. Behaviour Research and Therapy, 73, 58-66. https://doi.org/10.1016/j.brat.2015.07. 001

Brown, F. L., Whittingham, K., \& Sofronoff, K. (2014). Parental experiential avoidance as a potential mechanism of change in a parenting intervention for parents of children with pediatric acquired brain injury. Journal of Pediatric Psychology, 40(4), 464-474. https://doi.org/10.1093/jpepsy/jsu109.

Cheron, D. M., Ehrenreich, J. T., \& Pincus, D. B. (2009). Assessment of parental experiential avoidance in a clinical sample of children with anxiety disorders. Child Psychiatry and Human Development, 40(3), 383-403. https://doi.org/10.1007/s10578-009-0135-z

Cohrs, C. (2012). Efficacy of ACT components to increase effectiveness of behavioral parent training. Graduate thesis, University of South Florida.

Coyne, L. W., Low, C. M., Miller, A. L., Seifer, R., \& Dickstein, S. (2007). Mothers' empathic understanding of their toddlers: Associations with maternal depression and sensitivity. Journal of Child and Family Studies, 16(4), 483-497. https://doi.org/10.1007/s10826006-9099-9.

Coyne, L. W., McHugh, L., \& Martinez, E. R. (2011). Acceptance and Commitment Therapy (ACT): advances and applications with children, adolescents, and families. Adolescent Psychiatric Clinics of North America, 20, 379-399. https://doi.org/10.1016/j.chc.2011.01. 010.

Coyne, L. W., \& Murrell, A. R. (2009). The joy of parenting: An Acceptance and Commitment Therapy guide to effective parenting in the early years. New Harbinger.

Coyne, L. W., \& Thompson, A. D. (2011). Maternal depression, locus of control, and emotion regulatory strategy as predictors of preschoolers' internalizing problems. Journal of Child and Family Studies, 20, 873-883. https://doi.org/10.1007/s10826-011-9455-2.

Coyne, L. W., \& Wilson, K. G. (2004). The role of cognitive fusion in impaired parenting: an RFT analysis. International Journal of Psychology and Psychological Therapy, 3, 469-486.

Deković, M., Janssens, J., \& Van As, N. (2003). Family predictors of antisocial behavior in adolescence. Family Process, 42(2), 223-235. https://doi.org/10.1111/j.1545-5300.2003.42203.x.

Dumas, J. E. (2005). Mindfulness-based parent training: strategies to lessen the grip of automaticity in families with disruptive children. Journal of Clinical Child \& Adolescent Psychology, 34, 779-791. https://doi.org/10.1207/s15374424jccp3404_20.

Field, A. (2009). Discovering statistics using SPSS. Sage Publications.

Furman, W., \& Buhrmester, D. (1985). Children's perceptions of the personal relationships in their social networks. Developmental Psychology, 21, 1016-1024. https://doi.org/10.1037/0012-1649.21.6. 1016.

Gerris, J. R. M., Vermulst, A. A., van Boxtel, D. A. A. M., Janssens, J. M. A. M., van Zutphen, R. A. H., \& Felling, A. J. A. (1993). Parenting in Dutch families. University of Nijmegen, Institute of Family Studies.

Gerrits, L. A. W., Groenendaal, J. H. A., Deković, M., \& Noom, M. J. (1997). Handleiding van de Nederlandse versie van de Parental Dimensions Inventory (PDI). Universiteit Utrecht, Vakgroep Pedagogiek.

Gillanders, D. T., Bolderston, H., Bond, F. W., Dempster, M., Flaxman, P. E., Campbell, L., \& Masley, S. (2014). The development and initial validation of the cognitive fusion questionnaire. Behavior Therapy, 45(1), 83-101. https://doi.org/10.1016/j.beth.2013.09.001.

Greco, L. A., \& Eifert, G. H. (2004). Treating parent-adolescent conflict: Is acceptance the missing link for an integrative family therapy? Cognitive and Behavioral Practice, 11, 305-314. https://doi.org/10.1016/S1077-7229(04)80045-2.

Hayes, S. C., Luoma, J. B., Bond, F. W., Masuda, A., \& Lillis, J. (2006). Acceptance and Commitment Therapy: Model, processes and outcomes. Behavior Research and Therapy, 44(1), 1-25. https://doi.org/10.1016/j.brat.2005.06.006.

Hayes, S. C., \& Wilson, K. G. (1994). Acceptance and commitment therapy: Altering the verbal support for experiential avoidance. The Behavior Analyst, 17(2), 289-303. https://doi.org/10.1007/ BF03392677.

Jacobs, N., Kleen, M., de Groot, F., \& A-Tjak, J. (2008). Het meten van experiëntiële vermijding: De Nederlandstalige versie van de Acceptance and Action Questionnaire-II (AAQ-II). Gedragstherapie, 41, 349-361. 
Jacobson, N. S., \& Truax, P. (1991). Clinical significance: A statistical approach to defining meaningful change in psychotherapy research. Journal of Consulting \& Clinical Psychology, 59, 12-19. https://doi.org/10.1037/10109-042.

Johnston, C., \& Mash, E. J. (1989). A measure of parenting satisfaction and efficacy. Journal of Clinical Child Psychology, 18(2), 167-175. https://doi.org/10.1207/s15374424jccp1802_8.

Kowalkowski, J. D. (2012). The impact of a group-based acceptance and commitment therapy intervention on parents of children diagnosed with an autism spectrum disorder. Doctoral dissertation, Eastern Michigan University.

Matthijssen, D. (2012). Actief opvoeden: doen wat werkt voor jou en je kind. Hogrefe.

McCurry, C. (2009). Parenting your anxious child with mindfulness and acceptance. A powerful new approach to overcome fear, panic, and worries using acceptance and commitment therapy. New Harbinger Publications.

Moyer, D. N., \& Sandoz, E. K. (2015). The role of psychological flexibility in the relationship between parentand adolescent distress. Journal of Child and Family Studies, 24(5), 1406-1418. https://doi.org/10.1007/s10826-014-9947-y.

Murrell, A. R., Wilson, K. G., LaBorde, C. T., Drake, C. E., \& Rogers, L. J. (2008). Relational responding in parents. The Behavior Analyst Today, 9(3-4), 196-214. https://doi.org/10.1037/h0100659.

Ogles, B. M., Lunnen, K. M., \& Bonesteel, K. (2001). Clinical significance: History, application, and current practice. Clinical Psychology Review, 21(3), 421-446. https://doi.org/10.1016/ S0272-7358(99)00058-6.

Pinquart, M. (2017). Associations of parenting dimensions and styles with externalizing problems of children and adolescents: An updated meta-analysis. Developmental Psychology, 53(5), 873 https://doi.org/10.1037/dev0000295.

Raftery-Helmer, J. N., Moore, P. S., Coyne, L., \& Reed, K. P. (2016). Changing problematic parent-child interaction in child anxiety disorders: The promise of Acceptance and Commitment Therapy (ACT). Journal of Contextual Behavioral Science, 5(1), 64-69. https://doi.org/10.1016/j.jcbs.2015.08.002.

Shea, S. E., \& Coyne, L. W. (2011). Maternal dysphoric mood, stress, and parenting practices in mothers of Head Start preschoolers: The role of experiential avoidance. Child \& Family Behavior
Therapy, 33(3), 231-247. https://doi.org/10.1080/07317107. 2011.596004.

Slater, M. A., \& Power, T. G. (1987). Multidimensional assessment of parenting in single-parent families. In J. P. Vincent (Ed.), Advances in family intervention, assessment, \& theory (pp.197-228). Jai Press.

Tani, S., Kawai, E., \& Kitamura, K. (2013). ACT workshop for parents of children with developmental disabilities. Ritsumeikan Human Science Research, 28, 1-11.

Tiwari, S., Podell, J. C., Martin, E. D., Mychailyszyn, M. P., Furr, J. M., \& Kendall, P. C. (2008). Experiential avoidance in the parenting of anxious youth: Theory, research, and future directions. Cognition and Emotion, 22(3), 480-496. https://doi.org/10.1080/ 02699930801886599.

Tolan, P. H., Hanish, L. D., McKay, M. M., \& Dickey, M. H. (2002). Evaluating process in child and family interventions: Aggression prevention as an example. Journal of Family Psychology, 16(2), 220-236. https://doi.org/10.1037/0893-3200.16.2.220.

Whittingham, K., Sanders, M., McKinlay, L., \& Boyd, R. N. (2014). Interventions to reduce behavioral problems in children with cerebral palsy: An RCT. Pediatrics, 133, e1249 https://doi.org/ 10.1542/peds.2013-3620.

Williams, K. E., Ciarrochi, J., \& Heaven, P. C. (2012). Inflexible parents, inflexible kids: A 6-year longitudinal study of parenting style and the development of psychological flexibility in adolescents. Journal of Youth and Adolescence, 41 (8), 1053-1066. https://doi.org/10.1007/s10964-012-9744-0.

Wise, E. A. (2004). Methods for analyzing psychotherapy outcomes: A review of clinical significance, reliable change, and recommendations for future directions. Journal of Personality Assessment, 82 (1), 50-59. https://doi.org/10.1207/s15327752jpa8201_10.

Yap, M. B. H., \& Jorm, A. F. (2015). Parental factors associated with childhood anxiety, depression, and internalizing problems: A systematic review and meta-analysis. Journal of Affective Disorders, 175, 424-440. https://doi.org/10.1016/j.ja d.2015.01.050.

Fulton, J. J., Kiel, E. J., Tull, M. T., \& Gratz, K. L. (2014). Associations between perceived parental overprotection Experiential Avoidance, and Anxiety. Journal of Experimental Psychopathology, 5(2), 200-211. 